IBD

\title{
Evaluation of varicella immune status may be warranted in children with IBD
}

Screening for varicella immune status and appropriate vaccination may be required in children with newly diagnosed IBD, according to a new US study.

The course of the usually benign and common childhood disease varicella can be seriously affected by immunosuppression. Children with IBD often require immunosuppressive drugs to treat their disease. Following the introduction of a vaccine against varicella zoster virus (VZV), Susan Baker and her colleagues from the Women and Children's Hospital, Buffalo, NY, decided to investigate the immune status of 163 pediatric patients with newly diagnosed IBD. "We know that immunizations have a failure rate and that the risk of varicella in our immune compromised patients could be devastating", explains Baker.

A retrospective 5-year review of patient charts, the records of primary care physicians, the New York State Immunization Information System

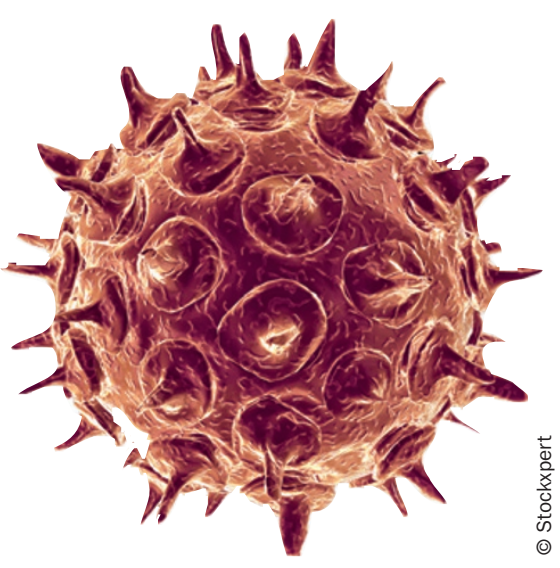

and parent immunization records was conducted. The group expected to find that at least $90 \%$ of their patients had antibodies against VZV and were protected by immunization or natural disease. $66 \%$ of patients had a history of varicella or had received vaccination. Measurable titers of antibodies against VZV were found in only $77 \%$ of patients.
"Since varicella is considered to be a universal childhood disease and VZV vaccine would have been readily available to individuals included in this study, we were surprised by the low number of patients with immunity," says Baker. "This is a wake-up call to assess children and adolescents with newly diagnosed IBD for varicella immunity. We cannot assume that our patients are protected, so we must measure antibody titers and vaccinate individuals when we find negative results. Hopefully vaccination can be done before immune suppression is initiated."

The team will now implement screening at their center for all newly dignosed IBD patients who will be treated with immunosuppressive drugs.

Rachel Thompson

Original article Ansari, F. et al. Varicella immunity in inflammatory bowel disease. J. Pediatr. Gastroenterol. Nutr. doi:10.1097/MPG.0b013e31821e1917 